\title{
Designing a Tablet-based Intervention to Support Self-checking for Melanoma
}

\author{
Matt Dennis \\ dot.rural RCUK Digital \\ Economy Hub \\ University of Aberdeen \\ Aberdeen AB24 5UA, UK \\ m.dennis@abdn.ac.uk
}

\author{
Peter Murchie \\ Academic Primary Care \\ University of Aberdeen \\ Aberdeen AB25 2ZD, UK \\ p.murchie@abdn.ac.uk
}

\author{
Judith Masthoff \\ Computing Science \\ University of Aberdeen \\ Aberdeen AB24 3UE, UK \\ j.masthoff@abdn.ac.uk
}

\author{
Kirsten A Smith \\ Computing Science \\ University of Aberdeen \\ Aberdeen AB24 3UE, UK \\ r01kas12@abdn.ac.uk
}

\begin{abstract}
In this paper, we describe the development of a tablet-based e-health app to help those previously treated for melanoma check their skin for recurrent or new melanomas. We present the results of a longitudinal pilot study, conducted in Scotland over six months with 20 patients previously diagnosed with melanoma. The app provided instructions on how to perform Total Skin Self-Examinations (TSSE), a set of high resolution photographs of their skin for comparison, and the facility to send information and photographs of suspicious skin changes directly to a nurse specialist. We investigate patients' experiences of this type of intervention and outline insights into the development of future e-health apps which encourage self-checking behaviours.
\end{abstract}

\section{Categories and Subject Descriptors}

J.3 [Life and Medical Sciences]: Medical Information Systems; H.5.2 [User Interfaces]: Prototyping

\section{Keywords}

eHealth Interventions, Melanoma, Self-Checking

\section{INTRODUCTION AND RELATED WORK}

Melanoma is one of the most common cancers in 15-34 year olds. More than $1 / 3$ of cases occur in people under 55 and, in the UK, it kills over 2,000 people every year[1]. The cost to the National Health Service in England alone was $£ 105.2$ million in 2008/9 [12].

The risk of malignant melanoma is between 8-15 times greater in people who have been diagnosed with a previous

Permission to make digital or hard copies of all or part of this work for personal or classroom use is granted without fee provided that copies are not made or distributed for profit or commercial advantage and that copies bear this notice and the full citation on the first page. Copyrights for components of this work owned by others than ACM must be honored. Abstracting with credit is permitted. To copy otherwise, or republish, to post on servers or to redistribute to lists, requires prior specific permission and/or a fee. Request permissions from permissions@acm.org. DH'15, May 18-20, 2015, Florence, Italy.

Copyright (C) 2015 ACM 978-1-4503-3492-1/15/05 ...\$15.00.

http://dx.doi.org/10.1145/2750511.2750518.

\author{
Susan Hall \\ Academic Primary Care \\ University of Aberdeen \\ Aberdeen AB25 2ZD, UK \\ s.hall@abdn.ac.uk
}

melanoma [2]. Early detection of these recurrences is thus a critical goal of follow-up programmes [13]. However, effectively delivering these follow-up programmes is problematic: many patients live far from a hospital, or otherwise find it difficult to arrange a follow-up visit. Recurrences and new primaries often occur between these consultations [13]. Thus it has been proposed that patients treated for cutaneous melanoma should be taught how to perform Total Skin Selfexaminations (TSSEs) and instructed to perform these at frequent intervals between appointments. TSSEs are likely to be an effective way of identifying potential recurrences patients treated for melanoma who detected their own recurrences had up to a $63 \%$ reduction in mortality $[4,8]$. It has also been found that skin self-examination can reduce the development of advanced disease and facilitate the early detection of recurrences by melanoma patients [3].

Clearly, a structured programme of TSSE would be beneficial for the detection of recurrences. Indeed, such training is possible $[9,11,14]$ and technology can effectively aid the training procedure [17]. However, patients are not consistently informed about the importance of self-checks, or trained on how to perform them [10, 7]. Once patients have learned about both the importance and method of TSSEs, performing self-checks and getting feedback from health professionals may help reinforce their confidence in self-checking and help sustain the behaviour [16]. Thus we propose that a clear and simple electronic guide to TSSE should be provided to patients to empower them to have confidence in their TSSEs.

However, even if patients can learn self-checking, this is likely to decline over time without an intervention to sustain their behaviour $[6,7]$.There is extensive evidence to suggest that mobile telephone and internet interventions can help promote health behaviour change (e.g. $[5,19,15])$, while there is some evidence to suggest that Apps (i.e. mobile or tablet applications) can be used to support a sustained health self-management strategy [20]. However, skin cancer apps that attempt to automatically identify melanoma risk are largely inaccurate [21]; therefore it is important that the patient's healthcare team are involved to check skin changes and offer swift treatment. We propose that an integrated tablet-based intervention might help promote a more 


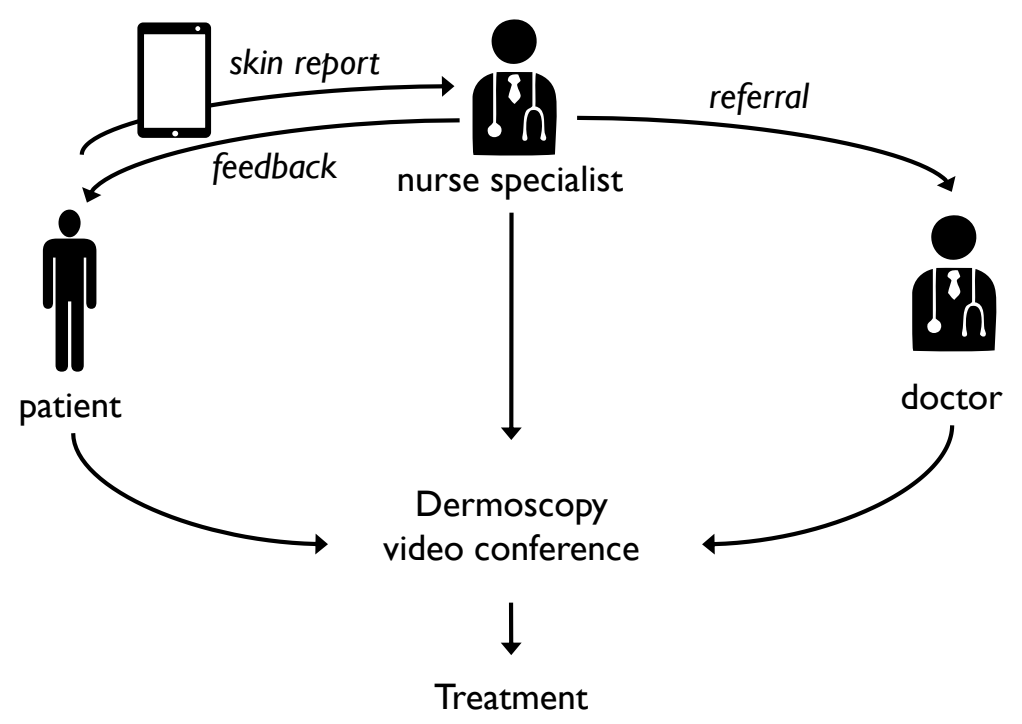

Figure 1: The ASICA intervention process. This shows the process when a patient reports a suspicious mole, which the nurse specialist thinks warrants further investigation.

sustained TSSE. This paper describes the development of a Skin-Checker app designed to promote TSSE for people treated for cutaneous melanoma.

\subsection{The ASICA Intervention}

The ASICA (Achieving Self-directed Integrated Cancer Aftercare) Skin-Checker app is sited within an intervention that aims to remove barriers between patients treated for melanoma and specialists in dermatology by enabling remote screening and diagnosis of skin changes.

Figure 1 shows the process of what happens within the ASICA Intervention when a patient discovers a skin change that they wish to report. First, the patient sends a skin report to the nurse specialist. The skin report contains a description of the change, where it is located, and a photograph of the change. If the nurse specialist thinks the change warrants further investigation, they will trigger an appointment with the patient and their local doctor (General Practitioner). At the appointment, the doctor and patient have a video conferencing session with the nurse specialist, using a dermoscope which can stream images to the nurse's computer. This allows diagnosis and potential treatment.

This process has several advantages over traditional procedures. Normally, the patient would have to travel to the nearest hospital with a consultant dermatologist to have their skin checked. In rural areas, this can be a considerable distance from the patient's home. For example, in Northern Scotland there are two such hospitals in Inverness and Aberdeen. For patients living on remote islands, this will involve a flight or ferry, taking considerable time and expense. With our intervention, the nurse can avoid the need for any further action if the skin change is not suspicious, and if they recommend further action, patients only need to visit their local doctor for a full consultation. Travel to the hospital is only required if the skin change requires biopsy or removal. This saves time and costs for both the patient and health service.
The ASICA Skin-Checker app was developed as part of this process. It had four key goals. The first was to enable patients to learn to perform a Total Skin Self Examination (TSSE). The second was to ensure that patients complete TSSEs regularly (e.g. once per month). An important part of performing a TSSE is the comparison of how the patients skin is changing over time; thus, the third goal was to provide a reference to previous skin appearance. The final goal was to enable patients to communicate any concerns directly with a health professional by sending a photograph and description of their skin.

\section{APP DEVELOPMENT}

\subsection{Prototyping Event}

To test the ASICA process and gain insights for the development of the app, an Experience Laboratory Event was held at the Glasgow School of Art's Centre for Design Innovation in Moray, Scotland. This facility allows the recreation of real-life situations. Six patient volunteers (unaffected by melanoma), a GP and a nurse specialist took part in the event. The patient volunteers were first shown a video produced by a Scottish melanoma support charity which provided detailed instructions on how to perform a TSSE. Afterwards, they performed a TSSE in a constructed "bedroom" with a non-functional prototype tablet.

The tablet displayed a mock up of an app which contained high-resolution photographs of a volunteer's skin for the whole body (the skin map). The app simulated the process of performing a full body skin check. We simulated scenarios where there were no problems detected, and some where a change was found which warranted investigation. Patients were guided through the process of first looking at their skin, then they could report a change if they wished. In the scenarios where a change was noticed, patients were asked to mark where the skin change was on an outline of a human body. They were then provided with a free-text 
box to describe the change, and the tablet then simulated a photo upload. Finally, the tablet simulated that this image had been sent on for investigation.

The next steps, following the discovery of a suspect mole were also simulated. Patients visited a constructed Doctor's surgery with the GP, and performed a video conference with the nurse specialist (who was situated in another part of the building). The event was filmed by a professional television production company. At the end of the event, volunteers were interviewed about the process and their opinions recorded.

\subsubsection{Findings}

The Process. The patient volunteers liked the process, and highlighted the advantages of reduced travel and waiting times for an appointment. They also liked the convenience of a next day appointment with their doctor rather than a consultant dermatologist and liked the idea that medical staff were monitoring their health more frequently. The follow up call should be within 24 hours, and the nurse should be clear, reassuring and allow them to answer questions. They wanted a text message to confirm the appointment time, if necessary. At the dermoscopy video conference appointment with patient, doctor and nurse specialist, they wanted a full screen video window to maximise the presence of the remote person and to allow eye-to-eye contact with all participants. Medical jargon should be avoided, and the remote specialist nurse should be introduced at the start of the call.

The App. Volunteers liked the idea of having skin map photos as reference for self-checking. The skin maps were of too low resolution to see marks in any detail, patients would prefer a larger skin map that they could zoom in on.

The video was seen as useful, however, although the patient volunteers understood the need for detailed instructions, they thought the video was too long. They felt that there was too much repetition, and the voice was too monotono They also stated that they would prefer two videos, one for each gender. Additionally, they felt that the video would be much more effective if it was integrated into the app (as opposed to a companion DVD), and was split into sections for different parts of the body.

Patient volunteers agreed that text-messages would make good reminders. They thought that the timing of the text message should be changeable per person, and suggested a time limit between the message and the expected report.

For the skin checking section of the app, the patient volunteers recommended that the checklists should be improved. In the prototype, the checklists simply stated to "check the skin for any changes" and they felt that they would benefit from more specific items, to ensure important steps are not missed. They wanted the checklist to be organized from head to toe as it reflected the natural order of a skin check. The skin-maps and video sections should be combined with the checklists, so that they can work on each section of the body independently. Volunteers thought that the checklist should still be completed and sent to the nurse specialist, even if they found no changes.

For the skin reports, volunteers recommended that they wanted instructions on how to crop and upload photos, and have the option to submit several photographs. The app should provide a list of common skin changes to choose from, rather than a simple text-box. They wanted to save photos so they could track changes and receive confirmation that the report was sent successfully. A telephone number should be given if there were any major concerns.

We concluded from the prototyping event that the process did not require any significant changes. However, the prototype app and instructional video required substantial improvements and integration.

\subsection{App Requirements}

Following the prototyping event, it was decided that the app had the following key requirements:

- The app should provide instructions on how to perform a TSSE

- The app should display high-resolution skin-maps for reference

- The app should allow patients to photograph suspect skin changes

- The app should report any suspect skin changes to a Nurse Specialist

- The app should record when all skin checks were performed

- the app should remind patients to perform a TSSE each month

\subsection{Form-Factor}

We had several choices on the form the app should take. Choices included: a web-application which ran on a standard PC, an app to run on a mobile phone, an app to run on a smart-camera, or an app to run on a tablet. We ruled out a standard $\mathrm{PC}$ as it would require a separate camera to provide images of sufficient quality for diagnosis, and would require patients to already have a computer that was sufficiently up to date. We ruled out mobile phones and smart cameras as the screen size is typically small (around 5 inches), and we were concerned that patients would find them too difficult to use. We decided that a tablet with a screen size of seven inches would be the best option, as this is large enough to be easy to use, but not so large that taking photographs of hard to reach body areas would become too cumbersome. We decided to develop for the Google Nexus 7 tablet (due to the relative ease of developing for Android), and provided each patient with a tablet to use in the trial.

\subsection{System Architecture}

We adopted a client-server architecture, the clients being the tablets which the patients interacted with. The server stored the patient's trial number, login credentials and device id (in hashed form), previous melanoma location and skin-maps. The server recorded whenever patients performed a TSSE, and the results. If the patient reported a change in their skin, this was stored and sent on to the nurse specialist by the server. If there were no changes reported by the patients after performing a TSSE, this was also stored.

\subsection{Security}

Due to the sensitive nature of the data, security concerns were very important. The server was hosted within a secure location at the University of Aberdeen, behind a corporate firewall, running an enterprise version of Linux. Administrator access to the server was only possible from within 


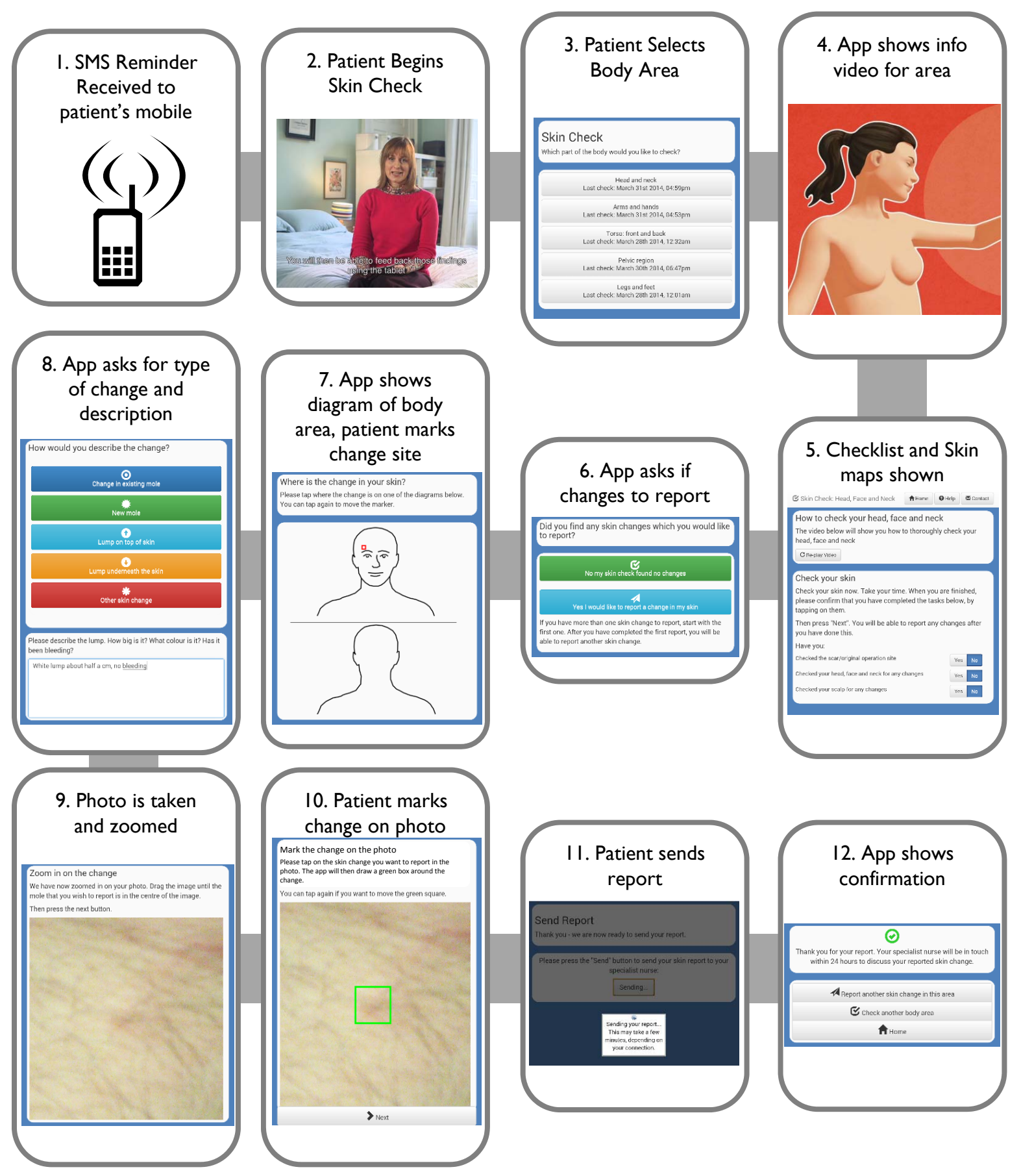

Figure 2: The Skin Check procedure used by the ASICA app when a patient wishes to report a change. 
the university network, on specified hosts. We stored the least amount of personally identifiable data possible; patients names and contact details were not stored, only a random trial number. The nurse specialist and one member of the team in Academic Primary Care held the mapping of patients' details to trial numbers offline in secure storage. The development team had no access to this information. The skin maps were stored on the server, and were personally identifiable in the cases where we stored the patients' faces (only if the site of their previous melanoma was on their face or head), and were all stored on an encrypted volume.

To use the app, the patients had to first log in using a username and password generated by the developer, logins had a time-out of 30 minutes of inactivity. To add an additional layer of security, the credentials only validated if they originated from the specific tablet that the patient was supplied with. That way, if a patient's credentials were compromised, access would still be denied. If a patient's tablet was lost or stolen, access could be immediately suspended by the team. As skin maps were not stored on the tablets (being delivered from the server each time), they would not be accessible in the event of a loss. Patients were provided with detailed instructions of what to do in the event of a loss of the tablet.

The server communicated with the tablets via the internet using a REST API over HTTPS, which encrypts all data in the transfer layer. The server would not transmit any data (other than processing logins) unless the patient had logged in from the correct tablet. The skin reports were stored in a database back-end, which was not accessible from any host other than the server itself. Skin reports were delivered in encrypted form to the nurse specialist using internal email on the university network only. The nurse specialist destroyed the skin reports after a follow up decision had been made.

The app itself was not available on any public app stores (such as Google Play), and was pre-installed by the developer on each tablet, to prevent hackers installing the app on rooted tablets (those flashed with a non standard operating system which allows access to the byte-code of installed apps) and attempting to reverse engineer the software.

The server was regularly monitored for any hacking attempts, and updated with the latest security patches as soon as they became available (e.g. for Heartbleed). If there was any suspicion that a hack was being attempted, access would be removed from all external hosts immediately.

\subsection{Instructions on how to perform a TSSE}

Based upon the findings of the Experience Laboratory, we commissioned 2 new sets of new videos which provided instructions on how to perform a skin check. We provided a set for each gender, and there were five videos in each set, one for each area of the body (head, neck and face; arms and hands; chest and back; pelvic region; and legs and feet). These contained a mix of a live action person describing how to perform the check, with an animated person (with a gender matching the patient's) showing the procedure.

\subsection{Skin Maps}

Prior to the trial, high-resolution photographs of a patient's skin were taken by the Medical Illustration department at the University of Aberdeen to use as their skin map. These provided a reference of all existing skin features for patients to compare their skin to in later checks. All patients were to have their neck, torso and limbs photographed. If the patient's original melanoma site was on the face or head, an additional skin map was to be taken of the head. Patients wore underwear when their pelvic area was photographed.

\subsection{Skin Check Procedure}

The procedure for performing a skin check using the app is shown in Figure 2. The app segregates the skin check into the five sections described above. The date and time of the last check for each area is shown to the user. Patients begin by selecting the area of the body that they wish to check. The app then shows the instructional video for this area. Patients then check their skin, and complete a checklist to confirm that they have completed this properly. The app then asks patients if they have any skin changes that they wish to report. If there is nothing to report, the app returns the patient to the area selection screen, and a skin check of "clear" is sent to a central server. If the patient wishes to report a change, the app asks them to select what sort of skin change they are reporting (new mole, change in existing mole, new lumps, etc.), and asks them to provide a short description of the change (size, location, colour etc.). Patients are then asked to take a photo of the change using the integrated camera on the tablet. After the patient confirms that the image is the one they want to send, the image is cropped, the location of the skin change marked and the report is sent to the central server. The app notifies the patient when this is successful. The server then sends the skin report to the nurse specialist for further investigation. The nurse specialist will then respond via telephone to the patient, and clincial processes begun.

\subsection{Skin Check Reminders}

The skin check reminders were not incorporated into the app itself. They were sent by a member of the team by e-mail or text message, or phone call if a long period of time had elapsed with no checks reported by the patient. This was due to a number of factors - we did not want to store personally identifiable data about the patient on the server (patients were only identified by their ID), and we were unsure that patients would notice reminders on the tablet itself, particularly if they had become disengaged with the process and left the tablet to run out of battery charge.

\section{LONGITUDINAL PILOT STUDY}

To test the ASICA intervention, we ran a pilot study over six months with patients previously diagnosed with melanoma. Each patient was provided with a Nexus 7 tablet for the duration of the trial.

\subsection{Patient Recruitment}

We recruited 20 patients from six General Practices located with a wide geographical spread in Northern and NorthEast Scotland, and were selected following a computerized search of medical records. All patients were previously treated for subcutaneous melanoma in the past nine years. Nine patients were male, and eleven female. Patients were aged between 37 and 83, diagnosed between the ages of 33 and 81. There was no restriction on the site of the melanoma on the body. Participants were selected according to the early stage of their cutaneous melanoma at diagnosis (i.e. Stage IA, IB, IIA). The risk of recurrence in this group is lower than later stage diagnosis; as such, these patients are 
supported by less intense follow-up regimes, making TSSEs more important [18]. We selected patients with varying distances from a hospital equipped with the facilities to check for melanoma. Four patients resided in a city (hospital close by), 14 patients resided in or near smaller towns and villages (within two hour's drive of a hospital), and 3 patients resided on more remote Scottish islands (requiring airlift or ferry to reach a hospital). Patients had to be able to attend one of two training sessions at the University of Aberdeen to take part in the trial.

\subsection{Patient Training}

We organized a training session for patients with all members of the ASICA team- two Computing Science researchers (acting as demonstrators), the Clinical Lead investigator and a researcher from Academic Primary Care. The Clinical Lead gave an overview of the project's aims and objectives at the start of the sessions. Patients were informed that this was a pilot study, and if they had any serious concerns which they felt required immediate medical attention, they were to contact their doctor. Similarly, if there was a problem with the app and they did not receive a response from the Nurse Specialist after sending a Skin Report, patients were instructed to contact their doctor.

The demonstrator connected their own tablet to a projector so that patients could follow the instructions on their own tablets. A second demonstrator walked round the patients and assisted them where necessary. Patients were shown how to turn the tablet on and off, how to charge the tablet, how to connect the tablet to a wireless network and how to use the touch screen. Subsequently, the demonstrator ran through a skin check using the app, including showing how to view the instructional videos and view their skin maps. Patients were shown how to use the camera to take photos of their skin, and all patients completed a sample skin-report. Patients were provided with an instruction manual for the tablet, an instruction manual for the app itself (including instructions on how to connect the tablet to their own wireless networks) and contact details for the relevant members of the team (for technical support and general queries about the trial itself), and the training sessions were concluded.

We gained several insights from the training sessions. Some patients struggled to use the touch screen, due to having shaky hands, reduced mobility in their fingers or simply never using one before. For those with reduced digital mobility, styluses were provided. Patients needed to be shown how to assemble the chargers for the tablets, as they come in parts in the box. As the Nexus 7 is of uniform colour and symmetrical appearance, patients did not always know which way up they were holding the tablet, which changes the position of the power button and volume controls. We also discovered that the standard Android Camera App was not particularly intuitive for those unfamiliar with modern smartphones and tablets. Patients struggled to access the menus within the camera app itself, which can be used to access the timer function and switch between the front and rear cameras.

\subsection{Study Procedure}

Following the training session, patients were sent home with their tablets and documentation. Patients were sent a reminders at monthly intervals, and their skin checking activity was monitored.

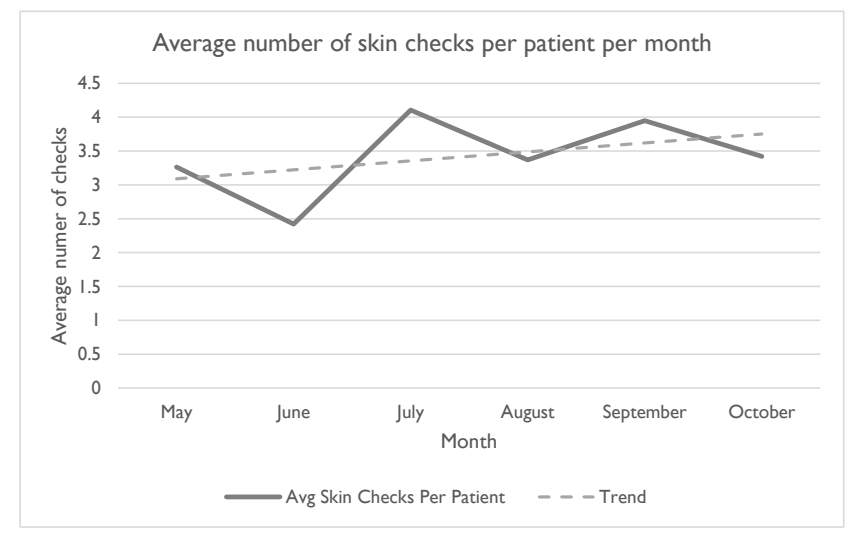

Figure 3: Average number of skin-checks per patient per month for the six months of the pilot study

\subsection{Outcomes}

Of the twenty patients recruited, one patient withdrew at the beginning of the study before they completed their first skin check.

Figure 3 shows the average number of skin checks per patient per month. As can be seen, the number of checks fluctuated throughout the trial, with fewer checks being performed in June, which coincides with the school summer holidays in Scotland. However, the overall trend is a rise in checks throughout the trial period.

We conducted unstructured interviews with patients at the end of the pilot study; fifteen were able to participate. There was a set of questions which the interviewer followed, however the interviewer adapted the questions depending on the respondents' answers. This allowed the patient to talk in some depth, choosing their own words. This helped us develop a real sense of the patient's opinions.

\subsubsection{Enabling patients to perform TSSEs}

Of fifteen, thirteen patients liked the skin checker app, and used it to perform their skin checks ("I'm very pleased with it because it's helping me [...] you feel in control or - yes you are looking after yourself!"). Of the two that did not, one patient stated that they still did not feel confident checking their own skin, preferring a medical professional to do it, and the other stated that they simply did not have time to do a full body check as they had a busy home life and only checked one area. Most patients said they thought that the video instructions on how to perform a skin check were good or excellent, and most preferred the animated figure performing the check rather than a real person. Reasons included that the animated person was less distracting, and they felt more appropriate as they had small children who they did not think should see a real naked body. Patients felt that the videos "make you more aware of your body", and "the study is helping me to remember to check all the parts and how to check".

Breaking the skin-check down into sections in the app was also well received. Patients felt it helped them to not forget certain body areas, and focus on areas which require more stringent checking (e.g. around the scar of their original melanoma site).

Seven patients required assistance from a partner or trusted person when performing skin checks, especially when check- 
ing their back. Four patients stated that they managed to do all their checking alone, as they had a good selection of mirrors.

Eight patients stated that the app was intuitive and easy to use, even for those not familiar with technology ("pretty self explanatory"; "easy for anybody to use"). The instruction manuals produced for the app were consistently well liked, two patients use them often to remind themselves how to use the app, and three patients stated that after the first few checks, they did not need the manual any more as they were comfortable with the app. Nine patients contacted technical support throughout the trial. Two calls were related to charging issues with the tablets, which were replaced. Five calls related to a bug within the user interface of the app, which was rectified. Three calls were related to forgotten passwords. One patient called to ensure that their 'clear' skin reports were being recorded.

\subsubsection{Performing Regular Skin Checks}

Patients were encouraged to perform their skin checks more often during the trial than they would have done without it ("I would say that it's encouraged me to [check] it's shown me the methodology of actually checking your skin carefully and making sure you are keeping your eye open. . . Which I'm not sure I would have done had I not been involved in this [trial]"; "before starting with the ASICA project, I probably wasn't really checking my skin that much at all. But since obviously I've been doing this, it's been much more regular, and I've been paying much more attention to it.").

Patients also found the reminders useful ("well I think that the fact that I was going through your monthly reminders actually kind of prompted me to keep a good look-out on any suspect skin areas"; "I appreciate all the help you've given me and the reminders to go and take responsibility for my own health."), and patients had set aside a particular day each month to perform their skin checks.

Eight patients responded positively when asked if they would continue checking their skin after the trial. Patients have become more aware of the need to check their bodies ("yes, yes I would and I mean I'm now [checking] after I've had a shower and I'm drying myself. .. I'm very, very much more aware of looking at my body"). Patients were even encouraging their family members to do the same ("My sister checks my niece who works abroad regularly").

\subsubsection{Skin Maps as a Reference}

Patients' experiences with the skin maps were mixed. Seven patients said that they were useful: "just to double check what they were like before" and "they are pretty handy as they can see historically where there are marks, new marks have come up and how the marks I have, have changed a little bit". Two patients found them confusing to use when they were checking skin areas using a mirror, as the skin map was then reversed. One patient stated that these should be re-created regularly to better track changes.

Four patients were less positive - one patient stating that "I don't find it useful at all". Others stated that they were not in enough detail, the Medical Illustration team had missed some areas of their body when they had the maps created, and that they would like them to be in a much higher resolution, with pan and zoom facilities. One patient stated that a video might be clearer than photographs. One pa- tient said that they did not like having photographs of their naked body (although underwear was worn) on the tablet as their granddaughter was helping them use it. One patient stated that they should be broken down into more images to make them easier to use.

\subsubsection{Sending Skin Reports}

Ten out of nineteen patients used the app to send skin reports to the nurse specialist. Overall, patients found it hard to take photos using the tablet. Four patients said that it's difficult to hold the tablet while taking photos of their own skin: "Because you actually have to hold the camera and also press the shoot button at the same time and it's quite difficult to hold the camera perfectly still whilst doing that"; several patients countered this by getting someone else to help to take the photos. One patient pointed out that one of the changes in skin that should be looked for - lumps would not appear well on a photo. A general problem for those who sent skin reports was that the photos were "not clear" or "blurred" (mentioned by five patients). Two patients reported sending a "back-up photograph" taken with their camera or mobile phone.

Two patients said that they liked being able to report a skin change directly to a nurse specialist. One patient reported a skin change, was referred to a GP, was diagnosed and advised on treatment without the need for a face-toface consultation. Another participant mentioned that they liked being able to send a photo if they were concerned and did not need to wait around at a clinic.

\section{CONCLUSION}

From this pilot study we have found that the majority of patients were receptive to an eHealth intervention to help them check their skin more regularly for recurrent or new melanomas. Our data shows that patients checked their skin at least as regularly at the start of the trial as at the end (if not more so), implying good adherence to TSSEs. Patients found the video instructions for performing a skin check useful - this helped them to learn to check their skin more thoroughly and regularly than before they participated in the trial. Most patients liked the skin map as a reference, although problems with their implementation sometimes limited their usefulness. Patients struggled to take clear photographs of skin changes, and required assistance. However, even if the original images submitted were too blurred for diagnosis, the nurse specialist was able to contact the patients and acquire a clearer image via email. Patients liked that there was a nurse specialist there to respond to any concerns that they had throughout the trial. One patient did discover a new melanoma via the app and specialist nurse, and received treatment.

\subsection{Insights for future eHealth apps}

We have identified several insights throughout this process which can be used for designing apps to support self-checking for certain medical conditions. Patients are receptive to interventions which include self monitoring and remote diagnosis. Patients appreciate the direct connection to a medical professional for reassurance of any issues they identify. A good quality physical user manual should be provided, with screenshots for every step. A telephone line for technical support should always be available, as many people are still unfamiliar with email, or don't check it frequently. Patients 
require photography training if this is to be used as part of an intervention. Bandwidth is still a common limitation, and any intervention should be responsive to this.

\subsection{Future Work}

Based on the results of this pilot study, we can improve the app to address some of the issues experienced by patients. The user-interface on the skin maps is to be improved, with better labelling of which body part the image is of (e.g. left arm, inner). Mid way through the trial, we were forced to reduce the resolution of the skin maps due to the slow speed of some patients' internet connections. This was reflected in the interviews, where patients stated that they were not in enough detail. The app can be improved to deal with this by allowing patients to specify if they have a high speed connection which supports larger resolutions. The quality of images received from the tablets can be improved by training patients to take clear photographs, including using natural light where possible, the timer function to remove the need to hold the tablet in the right place and press the shutter release button at the same time, and using the gridlines feature to make focusing easier. Following these improvements, we aim to take the ASICA intervention forward to a full randomized control trial.

\section{ACKNOWLEDGEMENTS}

This work was funded by the RCUK Digital Economy award to the dot.rural Digital Economy Hub, University of Aberdeen; award reference: EP/G066051/1. Process data about how ASICA performed during the pilot exercise and which does not identify patients may be available upon application to the first author.

\section{REFERENCES}

[1] Skin cancer key facts. Cancer Research UK Publications, 2014.

[2] Skin cancer risk factors. http://www.cancerresearchuk.org/, 2015.

[3] M. Berwick, C. B. Begg, J. A. Fine, G. C. Roush, and R. L. Barnhill. Screening for cutaneous melanoma by skin self-examination. Natl Cancer Institute, 88(1):17-23, 1996.

[4] K. M. Dalal, Q. Zhou, K. S. Panageas, M. S. Brady, D. P. Jaques, and D. G. Coit. Methods of detection of first recurrence in patients with stage i/ii primary cutaneous melanoma after sentinel lymph node biopsy. Ann Surg Oncol, 15(8):2206-2214, 2008.

[5] B. S. Fjeldsoe, A. L. Marshall, and Y. D. Miller. Behavior change interventions delivered by mobile telephone short-message service. American J. of preventive medicine, 36(2):165-173, 2009.

[6] K. E. Grady. Cue enhancement and the long-term practice of breast self-examination. Behav Med, 7(2):191-204, 1984.

[7] S. Hall and P. Murchie. Can we use technology to encourage self-monitoring by people treated for melanoma? a qualitative exploration of the perceptions of potential recipients. Supportive Care in Cancer, 22(6):1663-1671, 2014.

[8] P. R. Hull, N. G. Piemontesi, and J. Lichtenwald. Compliance with self-examination surveillance in patients with melanoma and atypical moles: an anonymous questionnaire study. Cutaneous Medicine and Surgery, 15(2):97-102, 2010.

[9] M. Janda, P. D. Baade, P. H. Youl, J. F. Aitken, D. C. Whiteman, L. Gordon, and R. E. Neale. The skin awareness study: Promoting thorough skin self-examination for skin cancer among men 50years or older. Contemp Clin Trials, 31(1):119-130, 2010.

[10] A. Körner, A. Coroiu, C. Martins, and B. Wang. Predictors of skin self-examination before and after a melanoma diagnosis: the role of medical advice and patient's level of education. Int Arch Med, 6(8), 2013.

[11] K. B. Lee, M. A. Weinstock, and P. M. Risica. Components of a successful intervention for monthly skin self-examination for early detection of melanoma: the 'check it out' trial. American Academy of Dermatology, 58(6):1006-1012, 2008.

[12] S. Morris, B. Cox, and N. Bosanquet. Cost of skin cancer in england. Eur $J$ Health Econ, 10(3):267-273, 2009.

[13] P. Murchie, M. Nicolson, P. Hannaford, E. Raja, A. Lee, and N. Campbell. Patient satisfaction with gp-led melanoma follow-up: a randomised controlled trial. Brit J Cancer, 102(10):1447-1455, 2010.

[14] S. A. Oliveria, S. W. Dusza, D. L. Phelan, J. S. Ostroff, M. Berwick, and A. C. Halpern. Patient adherence to skin self-examination: effect of nurse intervention with photographs. American J. of preventive medicine, 26(2):152-155, 2004.

[15] G. Phillips, L. Felix, L. Galli, V. Patel, and P. Edwards. The effectiveness of m-health technologies for improving health and health services: a systematic review protocol. BMC research notes, 3(1):250, 2010.

[16] J. K. Robinson, S. G. Fisher, and R. J. Turrisi. Predictors of skin self-examination performance. Cancer, 95(1):135-146, 2002.

[17] J. K. Robinson, R. Gaber, B. Hultgren, S. Eilers, H. Blatt, J. Stapleton, K. Mallett, R. Turrisi, J. Duffecy, M. Begale, et al. Skin self-examination education for early detection of melanoma: A randomized controlled trial of internet, workbook, and in-person interventions. Medical Internet research, 16(1), 2014.

[18] R. M. Turner, K. J. Bell, R. L. Morton, A. Hayen, A. B. Francken, K. Howard, B. Armstrong, J. F. Thompson, and L. Irwig. Optimizing the frequency of follow-up visits for patients treated for localized primary cutaneous melanoma. Clin Oncol, 29(35):4641-4646, 2011.

[19] T. Webb, J. Joseph, L. Yardley, and S. Michie. Using the internet to promote health behavior change: a systematic review and meta-analysis of the impact of theoretical basis, use of behavior change techniques, and mode of delivery on efficacy. Medical Internet Research, 12(1):e4, 2010.

[20] V. Williams, J. Price, M. Hardinge, L. Tarassenko, and A. Farmer. Using a mobile health application to support self-management in copd: a qualitative study. Brit J Gen Pract, 64(624):e392-e400, 2014.

[21] J. A. Wolf, J. F. Moreau, O. Akilov, T. Patton, J. C. English, J. Ho, and L. K. Ferris. Diagnostic inaccuracy of smartphone applications for melanoma detection. JAMA dermatology, 149(4):422-426, 2013. 